\title{
Infrared imaging techniques for the measurement of complex near-field antenna patterns
}

\author{
by J. Will ${ }^{1,2}$, J. Norgard ${ }^{1,3}$, C. Stubenrauch ${ }^{4}$, M. Seifert ${ }^{5}$ \\ 'University of Colorado, Colorado Springs, CO 80933, USA; ${ }^{2}$ SUN MicroSystems, Mountain View, CA \\ 94043, USA; ${ }^{3}$ US Air Force Academy, Colorado Springs, CO 80933, USA; ${ }^{4}$ National Institute of Standards \\ and Technology, NIST/Boulder, CO 80303, USA; ${ }^{5}$ US Air Force Rome Laboratory, Rome, NY 13441, USA
}

\begin{abstract}
This paper describes the application of "plane-to-plane" (PTP) iterative Fourier processing to infrared thermographic images of microwave fields to calculate the near-field and far-field patterns of radiating antennas. The PTP technique allows recovery of the phase by combining intensity (magnitude) measurements made on two planes, both in the radiating near field of the antenna under test. Starting with an estimate of the phase and the measured magnitudes. Fourier processing techniques are used to iteratively "propagate" between the planes to determine the correct phase distribution at each plane. We describe the technique and show excellent comparisons made between predicted and measured results.
\end{abstract}

\section{Introduction}

Thermographic measurements of microwave fields have been previously developed for mapping radiating field intensity patterns. It is necessary in most cases, however, to determine the complex (magnitude and phase) antenna near-field pattern. The purpose of this work was to develop and evaluate a technique for obtaining the necessary phase information from thermographic measurements. This work is described in more detail in [1, 2].

Direct measurement of phase information in standard hard-wired near-field antenna ranges requires the use of expensive vector measurement equipment and suffers from inaccurate measurements, particularly at the higher frequencies, due to errors such as temperatureinduced cable length changes. As a result, several algorithms have been proposed in recent years to retrieve phase information from phaseless (magnitude only) measurements.

Plane to plane (PTP) phase retrieval $[3,4,5]$ was specifically developed for near-field measurements of antennas. A closely related phase retrieval algorithm [6] has been successfully implemented by Yaccarino and Rahmat-Samii with a bi-polar planar hard-wired near-field measurement system using magnitude-only data measured over two planes separated by only $2.560 \lambda$ [7]. Further modifications and improvements to this technique have been carried out by Rahmat-Samii et al. [8] and Junkin et al. $[9,10,11]$. The uniqueness of the solution obtained from a plane-to-plane phase retrieval algorithm has been addressed by several authors, most notably Isernia, Leone, and Pierri [12]. This paper shows an application of PTP to the IR thermographic measurements of near-field antenna fields.

\section{Plane-to-Plane (PTP) phase retrieval}

\subsection{Introduction of PTP technique}

The setup used for PTP phase retrieval is shown in Fig. 1, and the phase retrieval process is illustrated in Fig. 2. First, various variables and constants are defined and an estimate of the magnitude and phase of the aperture field is made. This estimate is then "propagated" to measurement plane 1 by Fourier transformation. A convergence error is then calculated as 


$$
\xi=\frac{\sum|| A|-M|^{2}}{\sum M^{2}}
$$

where $M$ is the measured magnitude data and $|A|$ is the calculated magnitude data at each pixel location in the plane of interest. The calculated magnitude is then replaced with the measured magnitude with the calculated phase retained. These complex data are then propagated by Fourier techniques back to the original aperture plane. All data outside the antenna aperture are then truncated, and the truncated data are propagated to the second measurement plane. Again the convergence error is calculated and the calculated magnitude data replaced with the measured magnitude at plane 2 with the calculated phase retained, and these data are then propagated back to the aperture plane. At this point in the process, the change in the convergence error from the previous iteration is checked, and if the change in convergence error is less than a set tolerance, the iterations are halted. If, however, the change in convergence error is still sufficiently large, the iteration is repeated, starting with a truncation of data outside the antenna aperture.

The use of Fourier techniques for planar near-field to far-field transformations stems from the pioneering work of Kerns and his development of the plane-wave scattering matrix theory [13]. The planar near-field measurement was the first of the near-field techniques to be developed, verified, and implemented as an operational method of obtaining antenna parameters. An excellent review of the history of near-field antenna measurements is given by Yaghjian in [14], and a practical guide to planar near-field measurements and transformations is given by Newell in [15].

\subsection{Basic measurement setup}

A photograph of the measurement setup used in this work is shown in Fig. 3. A 36-element patch array antenna was used as the test specimen. The thermal paper, with its backing thermal insulator (poster board), is centered directly below the array antenna, oriented horizontally, and sitting on a wooden perimeter frame. The thermal imaging camera is shown at the bottom of the photograph.

The two measurement planes selected for the 36-element patch array antenna were at a distance of $32.4 \mathrm{~cm}$ and $45.0 \mathrm{~cm}$. Since the array operates at a frequency of $4 \mathrm{GHz}$, these distances were approximately $4.3 \lambda$ and $6 \lambda$. The exact distances were arbitrary, with the goals of being well outside the reactive near-field and having a plane separation of greater than one wavelength, but not so far apart as to result in a large difference in peak thermal paper temperatures.

\subsection{Simulations}

A set of simulations was performed before processing the thermally measured data. First, the array antenna was measured by the National Institute of Standards and Technology (NIST/Boulder) in their standard near-field antenna test range. The data provided by NIST on the array consisted of a $57 \times 57$ element matrix of field magnitude and phase data spaced 3.175 $\mathrm{cm}$ apart (about $0.4 \lambda$ ) in a plane $38.1 \mathrm{~cm}$ in front of the array. The near-field to far-field FFT processing method discussed above was then used to compute the magnitude and phase of the fields of the array at the two measurement planes selected for the IR thermal measurements $(32.4 \mathrm{~cm}$ and $45.0 \mathrm{~cm})$. The magnitudes of these data were then used as an initial simulation of the capabilities of the PTP algorithm.

Figure 4 is an overlay of the far-field pattern of the array as determined by the PTP algorithm (dashed ${ }^{*}$ ) and from the original NIST complex data (solid + ). As the figure illustrates, the 
agreement between the PTP-determined far-field pattern and the "real" far-field pattern of the array is excellent.

The PTP algorithm was then rerun with the NIST magnitude data truncated at amplitudes 20 $\mathrm{dB}$ or more below the peak as an estimate of the dynamic range of the thermal camera at the University of Colorado at Colorado Springs (UCCS). The result of this simulation is shown in figure 5. As illustrated in this figure, the PTP algorithm was able only to reconstruct the antenna main-lobe and provide an indication of the location of the first two side-lobes (but not the correct amplitudes for the side-lobes).

Obviously, the results from the simulations of the expected dynamic range from the UCCS thermal camera are only marginally useful; however, modern 12-bit digitizing thermal cameras, such as the camera available at Rome Laboratory, should have at least a $30 \mathrm{~dB}$ RF dynamic range. The results of the PTP algorithm applied to data with a $30 \mathrm{~dB}$ dynamic range are substantially better than those for data with only a $20 \mathrm{~dB}$ dynamic range, as shown in the simulation results of figure 6 . As shown in these simulations, data from thermograms collected with a camera having a $30 \mathrm{~dB}$ RF dynamic range should be adequate for the PTP algorithm to faithfully reproduce the far-field pattern of antennas such as the array tested in this paper. Future work will show the validity of this simulation, and additional work will focus on investigating the utility of the algorithm for other antennas, including some with lower sidelobes.

\subsection{IR thermogram results}

Actual IR thermograms were then taken over these same measurement planes. Direct comparison of the field magnitudes from the thermograms to the expected values based on the NIST measured data confirmed that the thermal measurements from the thermal camera at UCCS resulted in about 18 to $20 \mathrm{~dB}$ of usable dynamic range. The result of the PTP algorithm on these data are shown in figure 7 . The result of processing these thermograms is very encouraging as it is approximately the same as the $20 \mathrm{~dB}$ dynamic range simulation.

Another useful measure of the success of the PTP algorithm is a plot of the convergence error metric. Figure 8 shows an overlay of the convergence error metric of the PTP algorithm for the four cases discussed above (full range simulation, $30 \mathrm{~dB}$ dynamic range simulation, $20 \mathrm{~dB}$ dynamic range simulation, and UCCS thermogram data). Several observations can be made from this figure. First, the convergence metric settles to a stable value for each case in less than 40 iterations, which represents only 2 to 3 minutes of processing time on a 486DX2100 processor for these matrix sizes. Second, the convergence metric stays stable for many iterations (all runs were taken out for 200 iterations and all remained stable). Third, it appears that the value of the convergence metric is a usable measure of how well the algorithm was able to reconstruct the antenna far-field pattern. Since in actual practice, the antenna pattern will be unknown to the user (or the user would not be trying to measure it), the convergence metric may be very useful in determining the reliability of the PTP algorithm results.

\section{Summary/Conclusions}

In summary, the PTP iteration algorithm appears very well suited to the reconstruction of the holographic far-field pattern from thermographic measurements on two near-field planes. Additional research in the PTP technique should be pursued. First, a camera with greater dynamic range, such as the camera available at Rome Laboratory, should be used to verify the results of the $30 \mathrm{~dB}$ dynamic range simulation shown in this work. Furthermore, several antenna styles with different side-lobe amplitudes should be measured in order to build confidence in this technique. 


\section{Acknowledgments}

This work was partially sponsored by the US Air Force Office of Sponsored Research (AFOSA) and the US Air Force Small Business Innovative Research (SBIR) programs.

\section{REFERENCES}

[1] Will (J.). - Complex Antenna Pattern Measurements Using Infrared Imaging and Microwave Holography. Ph.D. Thesis, University of Colorado at Colorado Springs, April 1996.

[2] Will (J.), Norgard (J.), Stubenrauch (C.), MacReynolds (K.), Seifert (M.) and Sega (R.). Phase Measurements of Electromagnetic Fields Using Infrared Imaging Techniques and Microwave Holography. Proc. of the SPIE Aerosense Conference, Conference 2766, April 1996, p. 323-333.

[3] Ivanov (V.), Sivokon (V.) and Vorontsov (M.). - Phase Retrieval from a Set of Intensity Measurements: Theory and Experiment. Journal of the Optical Society of America, Vol. 9, No. 9, Sept. 1992, p. 1515-1524.

[4] Isernia (T.), Leone (G.), and Pierri (R.). - New Approach to Antenna Testing from Near Field Phaseless Data: The Cylindrical Scanning. IEE Proceedings, Vol. 139, Pt. H, Aug. 1989, p. 363-368.

[5] Bucci (O.), D'Elia (G.), Leone (G.) and Pierri (R.). - Far-field Pattern Determination from the Near-field Amplitude on Two Surfaces. IEEE Transactions on Antennas and Propagation, Vol 38, No. 11, Nov. 1990, p.1772-1779.

[6] Anderson (A.) and Sali (S.). - Diagnostics, Part I: Error Reduction Techniques. IEE Proceedings, Vol. 132, Pt. H., Aug. 1985, p. 291-298.

[7] Yaccarino (R.) and Rahmat-Samii (Y.). - Phaseless Near-Field Measurements Using the UCLA Bi-Polar Planar Near-Field Measurement System. 16th Annual Antenna Measurement Techniques Association Symposium, October 1994, p. 255-260.

[8] Yaccarino (R.) and Rahmat-Samii (Y.). - Phaseless Bi-polar Near-field Measurements: $A$ Squared Amplitude Interpolation/lterative Fourier Algorithm. 1995 Antenna Measurement Techniques Association 17th Meeting and Symposium, Williamsburg, Nov. 13-17, 1995, p. 195-200.

[9] Rizzo (C.), Junkin (G.) and Anderson (A.): - Near-field/Far-field Phase Retrieval Measurements of a Prototype of the AMSU-B Space-Borne Radiometer Antenna at $94 \mathrm{GHz}$. 1995 Antenna Measurement Techniques Association 17th Meeting and Symposium, Williamsburg, Nov 13-17, 1995, p. 385-389.

[10] Junkin (G.), Anderson (A.), Rizzo (C.), Hall (W.), Prior (C.) and Parini (C.).- Near-field/Farfield Phase Retrieval Measurement of a Prototype of the Microwave Sounding Unit Antenna AMSU-B at $94 \mathrm{GHz}$. Proc. of the ESA Workshop on Millimeter Wave Technology and Applications, ESA-WPP-098, ESTEC, Noordwijk, Netherlands, Dec. 5-7, 1995, p. 4.3.14.3.9.

[11] Trueba (G.), Junkin (G.). - A Numerical Beam Alignment Procedure for Planar Near-field Phase Retrieval. Electronics Letters, Vol. 31, No. 14, July 6, 1995, p. 1116-1117.

[12] Isernia (T.), Leone (G.) and Pierri (R.). - Phaseless Near-field Techniques: Uniqueness Conditions and Attainment of the Solution. Journal of Electromagnetic Waves and Applications, Vol. 8, No. 7, 1994, p. 889-908. 
[13] Kerns (D.). - Plane-wave Scattering-matrix Theory of Antennas and Antenna-Antenna Interactions. National Bureau of Standards Monograph 162, June 1981.

[14] Yaghjian (A.). - An Overview of Near-field Antenna Measurements. IEEE Transactions on Antennas and Propagation, Vol. AP-34, No. 1, Jan. 1986, p. 30-45.

[15] Newell (A.). - Planar Near-field Antenna Measurements. National Institute of Standards and Technology, Electromagnetic Fields Division Short Course Notes, March 1994.

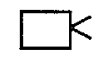

IR

Camera

Fig. 1. Schematic of PTP measurement setup.
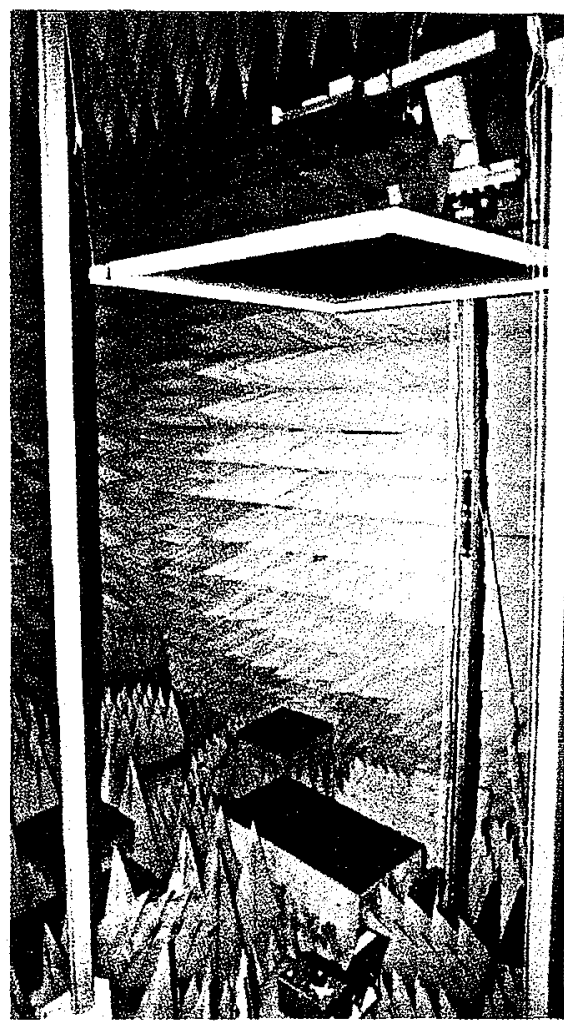

Fig. 3. Photograph of IR measurement setup.

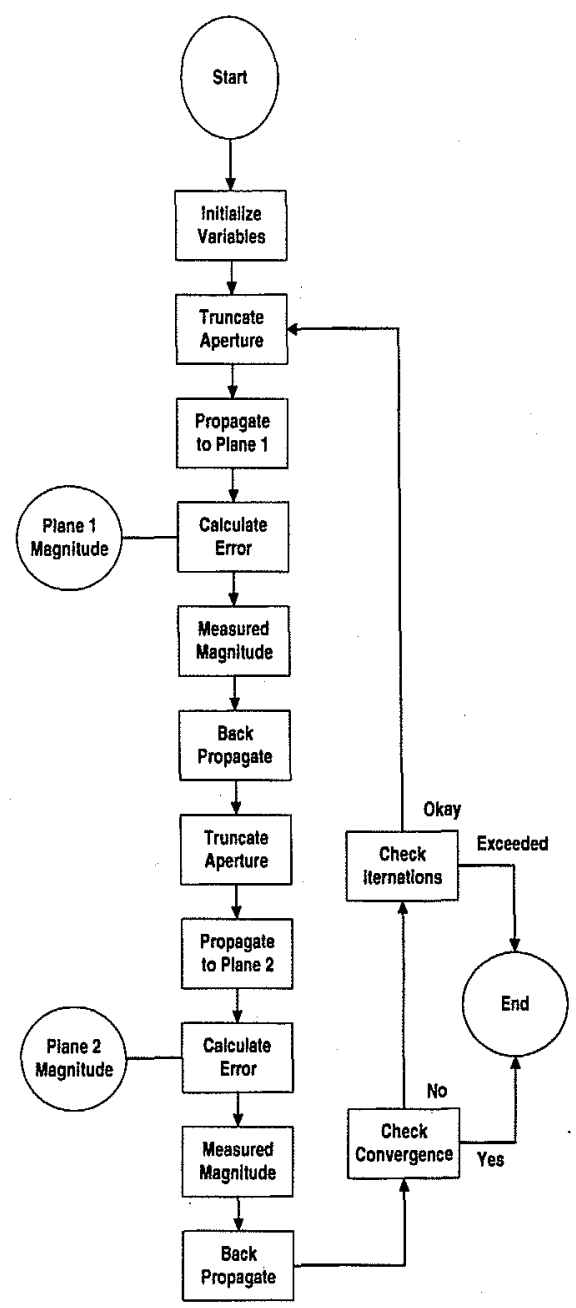

Fig. 2. PTP phase retrieval process. 
$d B$

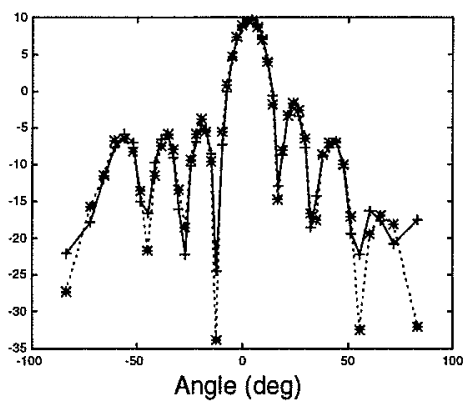

Fig. 4. PTP generated far-field from NIST magnitude data.

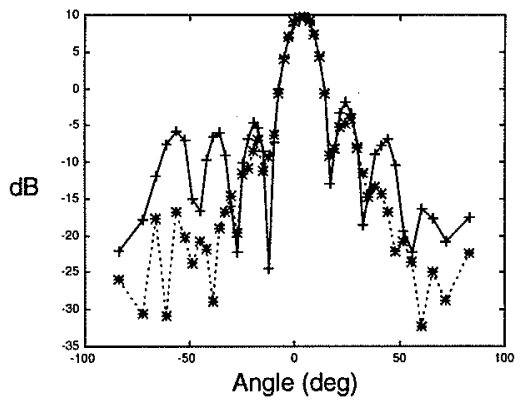

Fig. 5. PTP results using NIST magnitude data truncated to $20 \mathrm{~dB}$ dynamic range.

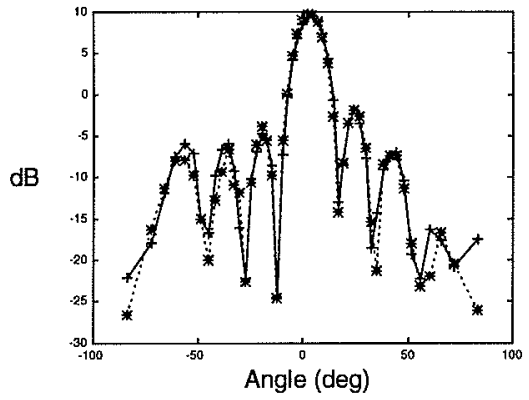

Fig. 6. PTP results from simulated $30 \mathrm{~dB}$ dynamic range data.

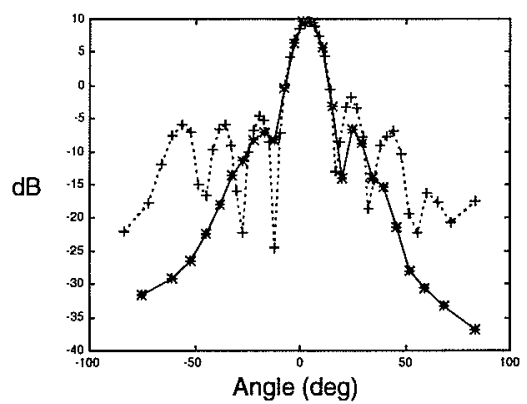

Fig. 7. PTP results for thermograms from the camera at UCCS.

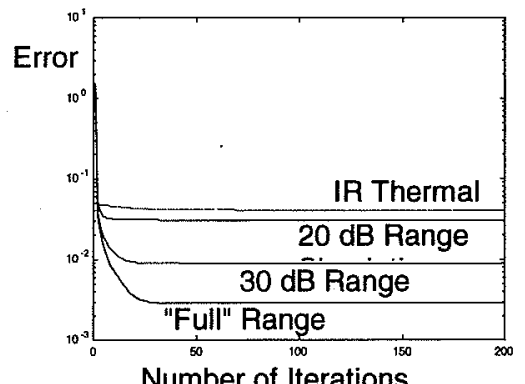

Fig. 8. Overlay of convergence error metrics for various PTP runs. 